\title{
Meeting Challenges of LTE Advanced through Small Cell Deployment
}

\author{
Juhi Bagaria and Hamid Shahnasser
}

\begin{abstract}
The paper focuses on how operators can meet challenges of LTE Advanced, LTE A through small cell deployment. LTE A is revolutionizing features such as Real time HD voice, video services and coverage with reduced cell drops. According to the performance targets defined by 3GPP LTE A will boost data rates from $150 \mathrm{Mbps}$ (LTE-Rel8) to 1Gbps. It will also ensure higher cell throughput, lower cost per bit, increased cell edge performance and upsurges of network efficiency. The answer lies in small cell deployment. The world is scaling down its parameters and so are the cell sites, allowing people to get more connected. Giant macro cell towers are being taken over with smaller units as also carriers are increasingly relying on small cell options. Small cells enhance cellular coverage and capacity in homes, enterprises, buildings and rural public areas. "They are low power wireless access points ranging from femto cells to microcells". This paper will review the enhancements that LTE Advanced will bring to the mobile network and the vital role that small cells play in realizing the advancement.
\end{abstract}

Index Terms-Heterogeneous networks, LTE advanced, small cells, small cell deployment strategies.

\section{INTRODUCTION}

There is an exponential increase in the mobile data traffic, and this presents challenges and problems, forcing the Network Operators to rethink their short and long term solutions that can relieve the data burden. Technologies such as HSPA, LTE and other broadband facilities including the world wide availability of smart phones and tablets, have exacerbated the data consumption. This is predicted to catch up pace even faster because of the innovation in machine to machine communications, where both wired and wireless technologies can communicate with various other devices using the same technology [1]. A recent study concluded that, $38 \%$ of phone calls and $50 \%$ of mobile data is consumed within the boundaries of home [2]. Small cells about the size of a router and capacity of 4 to 16 simultaneous users are being used in residential areas. But it is challenging to provide high speed service to every home in suburban and rural areas [2]. Thus large scale deployment of small cells is still in study phase. Although understanding why small cells are needed embarks a key step forward for operators in their effort for achieving high quality service provision. Section III starts the discussion with the standards set for LTE Advanced and different components that are taking LTE towards LTE Advanced. It outlines the technologies that form the backbone of LTE Advanced,

Manuscript received August 28, 2014; revised May 20, 2015.

Juhi Bagaria and Hamid Shahnasser are with San Francisco State University, United States of America (e-mail: juhi.bagaria@gmail.com, hamid@sfsu.edu). although most of them are still in study phase. Section IV of this paper describes in detail small cells and their deployment strategies.

\section{LTE ADVANCED}

LTE Advanced being combination of capacity and coverage provides a backbone for increasing relevance of small cells. The higher capacities, superior utilization factor and lower cost of subscriber handsets afforded by LTE technologies helps operators achieve a better return from their Capital and Operating expenditures. In a similar manner, IP-based backhaul networks will cut infrastructure costs by supporting call handoff between local cell nodes and concentrating traffic for transport across low-cost Ethernet links. Achieving these savings does, however, require more sophisticated base stations and backhaul links that can meet the precise timing requirements of the LTE A [1]. LTE Advanced in based on five technology components; Relay Nodes, Improved MIMO Schemes, Carrier Aggregation, Computed Multi-Point and Heterogeneous Networks. Weighing each technology component based on its advantages, the rest of this section will explain the vastly different features of LTE A. A phased approach towards LTE A roll-out in the market is provided taking every technology component in steps.

\section{A. Relay Nodes}

Radio waves cannot penetrate through the building walls easily, thus extending the coverage within homes, small offices and other indoor places becomes an issue and results in low signal strength to subscribers [3]. Thus certain transmitting nodes called as Relay Nodes are placed in the close vicinity of the user. This enhances both coverage and capacity. Unlike RF repeater, it not only re-broadcasts the signal but also demodulates the received signal, decodes and removes error and then re-transmits a new corrected signal. Relaying increases network density by increasing the number of e Node Bs. Small holes are filled and service outside the main area is provided using relays, thus it also boosts network coverage [4]. Since relays provide connectivity and increased data rates at the cell edges where there is low signal and more interference, they efficiently enhance cell edge performance. In addition relays are easy to install and hence reduce cost [5].

\section{B. Improved MIMO Schemes}

MIMO (Multiple Input Multiple Output) are being considered to enhance both peak and average spectral efficiencies. This technology enables transmission of multiple more data lines on multiple antennas. MIMO uses same resources for frequency and time but they are 
separated in reference signals. There are various schemes that are being researched for reaching out to multiuser interference minimization. LTE Advanced introduces higher order multiple input/multiple output supporting 8 antennas in downlink and 4 in the uplink [5]. Fig. 1 shows a $2 \times 2$ MIMO spatial multiplexing. In $8 \times 8$ or $4 \times 4$, multiplexing, the same concept is followed only with increased number of antenna. There are three types of schemes MIMO offers [5]. Multi-user MIMO strives for the best complexity performance trade-off. Single Site MIMO, will connect with single eNB with one or multiple UE devices. Cooperative MIMO: Cell edge user throughput is boosted. MIMO improves data rates without affecting transmitted power or bandwidth. In uplink more than one User Entity (UE) is scheduled to transmit in the same frequency. enB differentiates these signals by assigning orthogonal signals as reference. Hence, MIMO is proven a better technology in areas of higher Signal to Noise Ratio [5].

\section{Carrier Aggregation}

In order to meet peak data rates in LTE Advanced, bandwidth is increased from $20 \mathrm{MHz}$ (standard $\mathrm{BW}$ used in LTE) to $100 \mathrm{MHz}$ (standard BW set for LTE Advanced by ITU-R). Carrier Aggregation, a technology component present in LTE Advanced is a technique which addresses this challenge. Carrier Aggregation has been considered by various researchers, Mogensen, P. E. et al. [6] carefully reviews that in this method aggregated carrier, termed as component carrier is placed adjacent to each other [6]. Mogenesen et al. [6] categorizes three different scenarios for carrier aggregation. First Intraband contiguous, in this the component carriers are allocated in the same operating band and they are adjacent to each other. Second, Intra band non contiguous, the component carriers are again in the same subcarrier, but they are not adjacent any more. Thirdly, Inter band non contiguous, the component carriers are in different operating band. It is possible to widen the total bandwidth without widening contiguous spectrum.

\section{Computed Multipoint}

Another technology component present in LTE Advanced is Computed Multipoint (CoMP). It essentially enhances reception and transmission of signal, hence boosting throughput. An issue that LTE Advanced faces is in providing service to eNBs that are far from each other and placed at far ends geographically. Lopez-Perez et al., [7] reviews CoMP, and explains that it has two categories as that address the above challenge. First category as shown in Fig. 4 is Joint processing — when there is co-ordinaiton between multiple entities. That is, base stations are simultaneously receiving and transmitting to the UE. Second category is Beam Forming, when UE is transmitting with a single transmission or reception point. In this way, the cell is served by two more eNBs addressing the challenge to provide connectivity at the edge of the cell. Thus, these techniques are considered as promising candidates for efficient interference management to improve cell edge and system through put.

\section{E. Heterogeneous Networks}

In Heterogeneous Networks (HetNets), low power nodes are strategically placed thorough out the macro cell area. Thus the interference characteristics are different from homogenous network where only one type of cells is deployed [8]. Consequently, HetNet has completely different interference characteristics to a homogeneous deployment. To address this issue, Blankenship et al. [8] points out that careful consideration must be given to such differences when planning or optimizing the network. They take example of the following two scenarios:

Macro cell - Femto cell case: Femto cells serve a Closed Subscriber Group (CSG) and have a limited number of UEs that are registered to the femto cell. This scenario creates harsh interference conditions for UEs outside the CSG who are connected to the macro eNodeB while located in the vicinity of the femto cell. The positions of User Entity's (UEs) need special attention in network planning. The interference coming from the femto cell experienced by these UEs needs to be taken into account for coverage and, especially, capacity analysis.

Macro cell - Pico cell case: Pico cells serve an Open Subscriber Group (OSG) and can be accessed by any UE. From a UE perspective and for the purposes of coverage planning, there is no difference in the cell (re-)selection process. The UE selects the cell with the highest Downlink. In the UL however, a UE might be connected to a suboptimal cell. Home eNode B in a closed subscriber group creates a femto cell. Only a few registered UEs are allowed access to the group. This creates a mind boggling scenario for the macro UEs located in the HeNB coverage [9].

Proposed solution for this type of scenario is through Enhanced Inter Cell Interference Co-ordination (eICIC). Making efficient use of Frequency Division Multiplexing (FDM), cell layers can be distinguished on the basis of cross carrier scheduling [9]. LTE Advanced also supports Range Expansion (RE), where a cell selection bias (up to $6 \mathrm{~dB}$ ) is assigned to a pico cell. This is good for capacity planning as it can enhance throughput performance through an offloading effect towards the pico cell. However, range expansion also leads to harsh interference conditions for UEs in the expanded area, which needs to be reflected in the planning process [10].

\section{SMall Cell Depoloyment}

This section reviews various small cell deployment strategies existent in the industry and how each technology tries to overcome the challenges of backhauling. Backhauling comes into picture when connecting small cells to the macro network and then switching them on, where in the speed of data magnificently increases to 7.2 Mbps. Although the voice call increases from 8-12 kbps to only $48 \mathrm{kbps}$ for 4 users [11]. Thus, small cells are needed by the operators to relive the data and capacity demands in the constricted markets. They support temporary capacity and coverage and are easily deployable. [12] There are five different types of small cells existent in the industry [5]. Macro Cells, these are conventional cells. And their range is 5-6 kms, and around $46 \mathrm{dBm}$ power range. Pico Cells, they are low power operator installed towers. Range is $300 \mathrm{~m}$ or less with $23-30 \mathrm{dBm}$ power range. Femto Cells called as Home BS's or Home eNBs. Low cost, low power 
user deployed access points. Less than $50 \mathrm{~mm}$ and around $23 \mathrm{dBm}$ power range. Relays, operator deployed, route data from macro BS to end users. Remote radio heads - high power, low weight units. Mounted outside Macro BS connected through fiber. Enables distributed BS.

\section{Challenges in SMall Cell DePloyment}

There are numerous challenges that small cell deployment faces, primarily due to high quality service provided by micro cell networks. This paper focuses on backhauling challenge as the major hurdle encountered by mobile networks as reviewed by Weisi et al., [13]. This section reviews how small cell networks benefit mobile carriers to expand their coverage and capacity. This will include a discussion of the challenges involved with supporting small cells requirements across packet-based backhaul networks and other issues carriers encounter as they develop their 4G/LTE migration strategies.

\section{A. Small Cells Provide Enhanced Coverage}

For supporting the ever growing demand in mobile data traffic, operators are moving to data-offloading so as to make efficient use of micro cell spectrum. Small cells play important role in enhancing operator coverage. Thus they are elementary to tailoring around the LTE-A spectrum for better throughputs. Mobile users demanded networks with different radio access technologies, format of cells and then combine them in a uniform fashion; concept of HetNets was introduced in LTE Advanced. Thus although introducing eNBs reduces RAN congestion, but at the same time it makes the backhaul network congested.

\section{B. Co-ordination for Increased Spectral Efficiency}

Since adjacent cells use same frequency spectrum, interference between overlapping cells restricts users from proper throughput. Enhanced Intercell Interference Coordination restricts to radio resource management and thus attains high spectral efficiency. Both Time Division Duplexing (TDD) and eICIC need Base Stations to be synchronized for Uplink and Downlink. The problem hence arises if neighboring cells use same spectrum and the interference increases.

\section{Inter Cell Interference Co-ordination}

Ideal case is when the macro cell is far away and its coverage is poor. The Home e Node B reuses the frequency at low powers. Issue arises when the operator installs another macrocell in the area and it transmits at high power neat the same frequency. HeNB is not in the shadow anymore and this causes mutual inference. A conflict arises between which cell is going to reuse the frequency. Another issue is when an unauthorized phone is nearby, and if it's a closed subscriber group only authorized phones are allowed to attach to the femto cell. If there are multiple femto cels in the close proximity, they self-interfere. They need some sort of self-organizing structure [12].

\section{Small Cell Deployment Strategies}

Small cell deployment strategies are grouped into two categories based on their primary motivation and style of deployment [13]. First is Consumer or Enterprise self deployed and the second is Operator deployed small cells. Consumer deployed strategy is based only on Femto Cell Deployment and has no other sub categories. While Operator deployed small cells can be further sub categorized as Targeted capacity hot spots, Non targeted or peppered and Outdoor and indoor coverage non spots. This section discusses all strategies in detail and provides a solution to overcome the issue of backhauling.

\section{A. Femto Cells}

Femto cells being user deployed aim at improving both coverage and capacity in an area. These have a capacity of about 4 to 16 active users and they all can be served simultaneously. They are typically the size of a router, or cable/DSL modem, and serve the purpose of residential personal base station [14]. According to Saleh et al., [14] "They are a 'plug-and-play' technology with low transmit power, and are deployed in a random ad-hoc manner." There are three styles of deploying Femto Cells. Studies conducted by Saleh et al. [14], review that it is challenging and costly to install macro cell sites that can provide multimegabit per second coverage over the residential area. Also if the user moves away from the tower, shadowing, fading and multipath attenuation increases. An experiment conducted to compare the coverage with and without small cell installation showed that small cells provided a seamless smooth coverage with very less shadowing. Also it allowed users with broadband connection to have a connection equivalent to that of macro cell site since they didn't have to share bandwidth with multiple other users. Since femto cells use consumer based internet connection for backhauling, consumers have a choice whether to relax capacity or coverage. Thus applicable backhaul solutions include leased DSL, fibre and cable.

\section{B. Targeted Capacity Hot spots}

Shirakabe et al. [15] well summarizes this type of deployment style being implemented by the operator. Primary motivation involves enhancement of capacity through placement of hot-spot small cells in high demand density areas. They are deployed at the center of macro network. Thus, even though macro cell coverage is already present, hot spot small cells reduce sharing of mobile spectrum. It solves the problem of over congestion and thus interference caused by macro cell hot spots [15]. An ideal backhauling technique should be flexible enough to reach the hot-spot coverage location. Thus, places where a line of sight backhaul is available, point to point microwave or millimeter wave can serve the purpose. Otherwise a nonline of sight or fibre is considered. It is hard to come up with a single solution, because the smalls cells here provide capacity to already high coverage areas where the capacity demand is also sky rocketing.

\section{Non-Targeted Peppered}

Shirakabe et al. [15] further state that operator deployed small cells in a peppered fashion results in a flexible coverage, thus dousing capacity provided to customers. This technology specifically targets geo locations where cell location specification is more flexible. Thus, it benefits the most in terms of Quality of Experience in the areas where there is more need for enhancing the capacity. Non targeted 
peppered approach provides a flexible coverage therefore high capacity line of solutions can be researched for overcoming backhauling. Applicable solutions are using microwave and millimeter wave in cell sites.

\section{Outdoor and Indoor Coverage Non Spots}

Non-spots are locations where there is no existing macro coverage. It is very costly to install a macro cell site in such isolated places but a reduced cost small cell would suffice the need. Since there is no existing macro coverage in nonspots, available backhaul becomes complex. In outdoor-non spots, where it is hard to extend the coverage, applicable solutions can be through satellite, microwave or DSL line. On the other hand, in the indoor non-spots, existing internet service connection and LAN can be used to backhaul it to the network. Applicable solutions consist of providing service through fibre, DSL or microwave [15], [16]. Operators use a range of deployment technique for different small cell use cases. Based on above research I would like to propose that small cells are best utilized to provide high average throughput if not peak outputs. Thus, it appears small cells are best exploited where there is a need of high reuse of frequency spectrum and low interference, which bring us to femto cells. Section V discusses how femtosmall cells can improve capacity and coverage to address the standards set for LTE-Advanced.

\section{Combine Small Cells with LTE Advanced}

Subscribers' growing demand for advanced mobile data services represents both an opportunity and a challenge for wireless network operators. Upgrading networks to support 4G/LTE will allow them to serve new customers and offer advanced mobile services. Limited capital and the need to support their existing customer base make upgrades challenging. This section provides a quantitative assessment of how the deployment of small cells is addressing the challenges of LTE Advanced. In case of coverage, small cells provide basic service coverage and enterprise deployments. They are an alternative to RF repeaters. Thus addressing problem of RF coverage holes, hotspot coverage for $3 \mathrm{G}, \mathrm{LTE}, \mathrm{WiFi}$. Also in rural areas femto cells through HetNets address the same obstacle. In case of capacity, small cells provide localized capacity, while also increasing over all capacity with lower cost. Thus addressing the challenge of data load exceeding the capacity, limited macro expansion and cost.

Data offloading to femto cells, increases capacity of the network at an economical rate. Small cells established early successes with enhancing coverage and capacity of $3 \mathrm{G}$ networks in congested areas. This has led to network operators in believing that they will play a key role in strategizing and implementing heterogeneous networks. Inexpensive to procure and deploy, small cell networks are a cost-effective way to deliver the bandwidth needed to support advanced mobile data services in heavily congested areas. Another challenge that small cells address is of noncontiguous chunks of available spectrum. LTE advanced has carrier aggregation as the key technology that ensures carriers' existing spectrum to be aligned to deliver higher network throughput. Using enhanced Inter-cell Interference Coordination, small cells if deployed in LTE Advanced can alleviate radio interference. Since femto cells aim at optimizing spectral efficiency, the MIMO technology present in LTE A can suffice the need. Subscribers' growing demand for advanced mobile data services represents both an opportunity and a challenge for wireless network operators. Upgrading networks to support 4G/LTE will allow them to serve new customers and offer advanced mobile services. Limited capital and the need to support their existing customer base make upgrades challenging. This section provides a quantitative assessment of how the deployment of small cells is addressing the challenges of LTE Advanced. In case of coverage, small cells provide basic service coverage and enterprise deployments. They are an alternative to RF repeaters. Thus addressing problem of $\mathrm{RF}$ coverage holes, hotspot coverage for $3 \mathrm{G}$, LTE, WiFi. Also in rural areas femto cells through HetNets address the same obstacle. In case of capacity, small cells provide localized capacity, while also increasing over all capacity with lower cost. Thus addressing the challenge of data load exceeding the capacity, limited macro expansion and cost. Data offloading to femto cells, increases capacity of the network at an economical rate. Small cells established early successes with enhancing coverage and capacity of $3 \mathrm{G}$ networks in congested areas. This has led to network operators in believing that they will play a key role in strategizing and implementing heterogeneous networks. Inexpensive to procure and deploy, small cell networks are a cost-effective way to deliver the bandwidth needed to support advanced mobile data services in heavily congested areas. Another challenge that small cells address is of noncontiguous chunks of available spectrum. LTE advanced has carrier aggregation as the key technology that ensures carriers' existing spectrum to be aligned to deliver higher network throughput. Using enhanced Inter-cell Interference Coordination, small cells if deployed in LTE Advanced can alleviate radio interference. Since femto cells aim at optimizing spectral efficiency, the MIMO technology present in LTE A can suffice the need.

\section{CONCLUSION}

Small cells can be deployed to boost capacity in congested locations and provide coverage in areas such as urban canyons, where macrocells cannot reach. These lowpower, compact devices are equipped with integrated networking capabilities and can be housed in a variety of specialized packages which allow them to be easily integrated into an urban landscape. Small cells help wireless carriers improve the capacity and coverage of their $3 \mathrm{G}$ networks while enabling a cost-effective incremental upgrade path to 4G/LTE services. Small cell networks can be used to add capacity in congested areas and help extend the network's coverage to urban canyons and indoor environments where traditional macrocell infrastructures cannot reach. The predicted demand for data, coverage and capacity was reviewed. It was noted that there is a significant growth in the use of small cells. The industries are interested in providing backhaul solutions for operators who want to focus on improving service to mobile data subscribers. As with any technology prediction, this technology is also a combination of trial, error and intuition 
fueled by research. In the foreseeable future, it is safe to say that small cell technology will fill the gap between LTE and LTE- Advanced.

\section{REFERENCES}

[1] Web. [Online]. Available: http://www.actix.com/lte-advancedimpact.html

[2] J. Weitzen, M. Li, E. Anderland, and V. Eyuboglu, "Large-scale deployment of residential small cells," in Proc. the IEEE, no. 99, pp. $1-14$.

[3] Web.

[Online].

Available:

http://www.qualcomm.com/solutions/wirelessnetworks/technologies/lte-advanced

[4] S. Landstrom, H. Murai, and A. Simonsson, "Deployment aspects of LTE Pico nodes," in Proc. 2011 IEEE International Conference on Communications, June 5-9, 2011, pp. 1-5.

[5] R. Wireless, "Dealing with density, the move to small cell architectures," June 2012.

[6] P. E. Mogensen, T. Koivisto, K. I. Pedersen, I. Z. Kovacs, B. Raaf, K. Pajukoski, and M. J. Rinne, "LTE-Advanced: The path towards gigabit/s in wireless mobile communications," in Proc. 1st International Conference on Wireless Communication, Vehicular Technology, Information Theory and Aerospace \& Electronics Systems Technology, May 17-20, 2009, pp. 147-151.

[7] A. Prasad, O. Tirkkonen, P. Lunden, O. N. C. Yilmaz, L. Dalsgaard, and C. Wijting, "Energy-efficient inter-frequency small cell discovery techniques for LTE-advanced heterogeneous network deployments,' Communications Magazine, vol. 51, no. 5, May 2013.

[8] Y. W. Blankenship, "Achieving high capacity with small cells in LTE-A," in Proc. 2012 50th Annual Allerton Conference on Communication, Control, and Computing, Oct. 1-5, 2012, pp. 16801687.

[9] Cambridge Broadband Networks, "Small cells deployment strategies and best backhaul practices," August 2012.

[10] W. S. Guo, S. Y. Wang, X. L. Chu, J. Zhang, J. M. Chen, and H. Song, "Automated small-cell deployment for heterogeneous cellular networks," Communications Magazine, vol. 51, no. 5, pp. 46-53, May 2013.

[11] Cambridge Broadband Networks, "Small cell deployment strategies and best practices," August 2011.

[12] Y. S. Soh, T. Q. S. Quek, M. Kountouris, and H. D. Shin, "Energy efficient heterogeneous cellular networks," IEEE Journal on Selected Areas in Communications, vol. 31, no. 5, pp. 840-850, May 2013.
[13] J. Weitzen, L. Mingzhe, E. Anderland, and V. Eyuboglu, "Largescale deployment of residential small cells," Proceedings of the IEEE, vol. 101, no. 11, pp. 2367-2380, Nov. 2013.

[14] A. B. Saleh, S. Redana, B. Raaf, and J. Hamalainen, "Comparison of relay and Pico eNB deployments in LTE-advanced," in Proc. 2009 IEEE 70th Vehicular Technology Conference Fall (VTC 2009-Fall), Sept. 20-23, 2009, pp. 1-5.

[15] M. Shirakabe, A. Morimoto, and N. Miki, "Performance evaluation of inter-cell interference coordination and cell range expansion in heterogeneous networks for LTE-Advanced downlink," in Proc. 2011 8th International Symposium on Wireless Communication Systems, Nov. 6-9, 2011, pp. 844-848.

[16] G. W. Juette and L. E. Zeffanella, "Radio noise currents n short sections on bundle conductors," presented at the IEEE Summer Power Meeting, Dallas, TX, June 22-27, 1990.

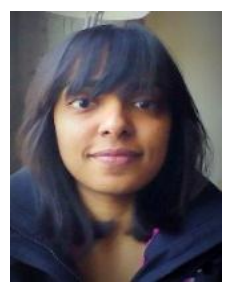

Juhi Bagaria was born on July 27, 1989. She has received her bachelors in electrical engineering degree from the Institute of Technology and Management, Delhi, India. She received her MS degree in embedded electrical and computer systems from San Francisco State University, USA.

Her areas of research and interest include wireless systems, computer networking, embedded systems and software development. She is an active member of the Bay Area Engineers and regularly contributes to open source projects as a developer evangelist.

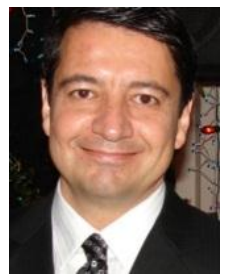

Hamid Shahnasser received the B.E. degree in electrical engineering from McGill University, Montreal, received his MS degree in electrical and computer engineering from Carnegie-Mellon University and his Ph.D. degree from Drexel University Pennsylvania.

$\mathrm{He}$ is currently a professor in electrical and computer engineering at San Francisco State University as well as the graduate program coordinator. His areas of interest are communication networks and computer systems. Dr. Shahnasser has been a research faculty consultant of NASA Ames Research Center since 1990 and has been collaborating on several research grants with that organization since then. He has received grants from NSA, Department of Education, National Science Foundation and various private companies carrying out research in the area of his interest and expertise. 